\title{
PAISAJE LINGÜÍSTICO INSTITUCIONAL EN EL ÁREA DIALECTAL BONAERENSE: LA PROMOCIÓN DE LA ENSEÑANZA DE LENGUAS
}

\author{
Institutional linguistic landscape in Buenos Aires dialectal area: \\ the language teaching promotion
}

\author{
YOLANDA Hipperdinger* \\ UNS / CONICET \\ yhipperdinger@uns.edu.ar
}

\author{
Palabras clave \\ paisaje lingüístico \\ institucional; \\ instituciones de \\ educación superior; \\ enseñanza de lenguas; \\ estrategias de promoción
}

\section{Keywords}

institutional linguistic

landscape;

higher education

institutions; language

teaching;

promotion strategies

\section{RESUMEN}

El estudio del llamado paisaje lingüístico, esto es, la distribución socio espacial de los usos de las diversas lenguas y variedades lingüísticas -muchas veces en contacto-, es aún de desarrollo incipiente en la Argentina. La mayor parte de las investigaciones que se vienen desarrollando corresponden al área dialectal bonaerense, y en la misma área se llevó adelante la investigación cuyos resultados se presentan en este artículo. No obstante, y frente a un interés prácticamente exclusivo por los espacios exteriores, nos ocupamos aquí de espacios de acceso público pero arquitectónicamente interiores: abordamos el paisaje lingüístico que suele llamarse institucional, enfocando la promoción de la enseñanza de lenguas extranjeras en dos instituciones de educación superior (universidades de gestión pública) ubicadas en la ciudad de Bahía Blanca, referencial para el área dialectal referida. Registramos y analizamos el modo en que se promociona en esas instituciones el ofrecimiento de clases extracurriculares de lenguas extranjeras: por un lado, precisamos para qué lenguas se las ofrece, y por parte de qué emisores; por otro lado, exploramos las estrategias de promoción que los distintos actores emplean, de acuerdo con la lengua de que se trata.

\begin{abstract}
The study of the so-called linguistic landscape, that is, the socio-spatial distribution of the uses of the various languages and linguistic varieties - many times in contact-, is still in incipient development in Argentina. Most of the researchs that are being developed correspond to the Buenos Aires dialectal area, and the one whose results are presented in this article was carried out in the same area. Nevertheless, and differentiating ourselves from the practically exclusive interest in outdoor spaces, we deal in this paper with spaces with public access but architecturally interior: we approach the linguistic landscape that is usually called institutional, focusing the promotion of foreign language teaching in two higher education institutions (universities with public management) located in the city of Bahía Blanca, a reference point for the referred dialectal area. We register and analyze the way in which the offering of extracurricular foreign language classes is promoted in these institutions: on the one hand, we specify for which languages they are offered, and by which senders; on the other hand, we explore the promotion strategies that the different actors use, according to the language in question.
\end{abstract}




\section{Introducción}

El conocimiento de las variedades dialectales del español de la Argentina fue impulsado extraordinariamente por la publicación de la obra colectiva coordinada por María Beatriz Fontanella de Weinberg, El español de la Argentina y sus variedades regionales (2000), que, a ya más de dos décadas de su elaboración, constituye aún la visión de conjunto más completa disponible. En el proyecto de esa obra, Fontanella de Weinberg siguió de cerca la división en áreas dialectales oportunamente pergeñada por el aporte de Berta Vidal de Battini (1964), fundamental para la dialectología del país, con la sola innovación de dividir la que esta autora había denominado región litoral en tres regiones diferenciadas: la bonaerense, la litoral propiamente dicha y la patagónica, que se sumaron a las regiones nordeste, noroeste, central y cuyana. Para asegurarse de reunir conocimiento de detalle y calidad sobre el español de la Argentina, Fontanella de Weinberg convocó a destacadas lingüistas de las distintas áreas dialectales del país para la elaboración de sus descripciones, todas ellas con orientación sociolingüística: Nélida Donni de Mirande en la región litoral, Elena Rojas Mayer en la noroeste, Magdalena Viramonte de Ávalos en la central, Liliana Cubo de Severino en la cuyana y, en la nordeste, Inés Abadía de Quant, a quien tan merecidamente se homenajea en este número.

Aunque sin generar todavía ninguna nueva summa, el conocimiento de las peculiaridades de las distintas variedades dialectales argentinas, tanto formales como funcionales, ha avanzado notoriamente desde aquella, e incluso nuevos objetos de atención han pasado a ocupar a los estudiosos. Una de las líneas de investigación de desarrollo más reciente, y todavía incipiente, atañe a un objeto que fue construido como tal sobre el cambio de milenio: el llamado paisaje lingüístico (linguistic landscape en inglés, la lengua de las contribuciones fundantes ${ }^{1}$ y de la mayor parte de las publicadas), esto es, la distribución socioespacial de los usos de las diversas lenguas y variedades lingüísticas, muchas veces en contacto. ${ }^{2}$ El estudio del paisaje lingüístico argentino está dando sus primeros pasos, pero es de prever que crezca y permita disponer de descripciones para las distintas áreas dialectales del país, de modo que cuando vuelva a publicarse una comprehensiva visión de conjunto pueda abarcar también esta peculiar manifestación de los usos del lenguaje: la de una exbibición de producciones lingüísticas que transmiten mensajes que dialogan entre sí, tanto como remiten a las condiciones y motivaciones que les dieron origen y se mantienen asequibles a potenciales destinatarios futuros. ${ }^{3}$

1. El aporte considerado propiamente fundacional para los estudios del paisaje lingüistico es el de Rodrigue Landry y Richard Bourhis (1997). Aunque estos autores reconocen como antecedentes solamente los trabajos de los planificadores lingüísticos Albert Verdoot (1979) y Jean-Claude Corbeil (1980), en el marco -respectivamente- de los conflictos políticolingüísticos de Bélgica y Canadá (Landry y Bourhis, 1997, p. 24), suelen valorarse como antecedentes también otras contribuciones, como la de Rosenbaum et al. (1977). Como parte del "primer momento" en la constitución de los estudios sobre paisaje lingüístico cabe referir, particularmente, los aportes de Scollon y Wong Scollon (2003) y Griffin (2004).

2. Discusiones relativas a la conceptualización del paisaje lingüistico pueden verse, por ejemplo, en Gorter (2006), Shohamy, Elana y Durk Gorter (2009), Pons Rodríguez (2012), Blommaert (2013), Van Mensel, Vandenbroucke y Blackwood (2016) y Wee (2021). Las principales ampliaciones de ese recorte objetual -ambas hoy con difusión en el campo- son las de paisaje semiótico y paisaje virtual; al respecto pueden verse, respectivamente, Jaworski y Thurlow (2010) e Ivković y Lotherington (2009).

3. En el paisaje lingüístico, como lo señalan Blommaert y Maly (2014,p. 4), "[s]igns point towards the past, to their origins and modes of production[,] (...) point towards the future, to their intended audiences and preferred uptake[, and] point towards the present, through their 'emplacement"' (resaltado original). 
Si bien, como dijimos, el paisaje lingüístico recién ha comenzado a estudiarse en la Argentina, existen ya aportes relativos a varias áreas dialectales, aunque la mayor parte de los trabajos que se han desarrollado hasta el momento corresponden al área bonaerense. ${ }^{4} \mathrm{~A}$ esta última corresponde también la circunscripción territorial que hemos seleccionado para la investigación que se informa en este artículo, que aborda paisaje lingüístico de la ciudad sudbonaerense de Bahía Blanca, referencial para el área desde los destacados estudios de Fontanella de Weinberg (i.a. 1979). En este caso no nos ocuparemos del paisaje lingüístico exterior, como es corriente, sino que nos adentraremos en una de las más novedosas expansiones del campo, atendiendo al paisaje lingüístico de espacios de acceso público pero arquitectónicamente interiores: el paisaje lingüístico que suele llamarse institucional, que enfoca la mensajería exhibida en instituciones educacionales, de salud, etc. Esta nueva perspectiva puede contribuir notoriamente a la comprensión de la dinámica de las instituciones, así como a la de las estrategias de comunicación pertinentes y las políticas lingüísticas (explícitas o no) que se hallan implicadas. ${ }^{5}$

\section{La investigación: oferta y paisaje lingüísticos en instituciones universitarias}

En la línea de indagaciones referida, presentaremos en este artículo los resultados de un acercamiento al paisaje lingüístico de instituciones de educación superior universitaria de gestión pública en Bahía Blanca, diseñado para registrar y analizar el modo en que se promociona en ellas el ofrecimiento de clases de las lenguas que, en el ámbito educacional, se denominan extranjeras. ${ }^{6}$

En el paisaje lingüístico de esas instituciones, tal ofrecimiento convive con el que hacen otros emisores (centros privados de enseñanza de idiomas, profesores particulares), con los mismos estudiantes como destinatarios. En este artículo nos ocuparemos de precisar para qué lenguas se publicitan clases en cada una de las direcciones citadas (la oficial y la de otros emprendimientos, que se corresponden con las dimensiones comúnmente denominadas top-down y bottom-up $)^{7}$ y de analizar los beneficios que en cada caso se invocan en relación con el aprendizaje de cada una de las lenguas cuya enseñanza se promociona.

Los datos sobre los que trabajamos corresponden al relevamiento realizado durante las semanas iniciales de cada cuatrimestre lectivo, en el año 2018, ${ }^{8}$ en la Facultad Regional Bahía Blanca de la

\footnotetext{
4. Por los desarrollos sobre el área bonaerense pueden consultarse, principalmente, Bonnin y Unamuno (coords.) (2019) y Hipperdinger (ed.) (2019). Una muestra del comienzo de los estudios en otras áreas dialectales del país puede verse en los trabajos reunidos para el Simposio "Lenguas promovidas, lenguas silenciadas: el estudio del paisaje lingüístico" del XVII Congreso de la Sociedad Argentina de Estudios Lingüísticos (2021).

5. Pueden verse al respecto, entre otros, los aportes de Hanauer (2010), Gorter y Cenoz (2015), Rámila Díaz (2015) y Albizu Echeipia (2016).

6. Sobre la denominación (y las lenguas preferidas) pueden consultarse los trabajos de Pozzo (2009) y Bein (2012).

7. Respecto de estas dimensiones es señera la contribución de Ben-Rafael, Shohamy, Hasan Amara y Trumper-Hecht (2006, p. 14). La vigencia de su consideración se demuestra, por ejemplo, en Leimgruber (2019).

8. Una única excepción se ha hecho a ese período, en el caso del inicio tardío (a mediados del primer cuatrimestre) del ofrecimiento de clases de chino por la Universidad Nacional del Sur (v. Fotografía 1).
} 
Universidad Tecnológica Nacional y en tres de los principales edificios del complejo de la Universidad Nacional del Sur: por un lado los de más tradición, que albergan el Rectorado y la Biblioteca Central, y por otro lado el edificio que corresponde al Departamento de Humanidades, que concentra los cargos docentes sostenidos por esa Universidad para la oferta de enseñanza de lenguas. ${ }^{9}$

La exigencia de conocer otras lenguas además de la propia (cuando menos, lenguas de amplio uso vehicular) suele estar inscripta en los diseños de las carreras universitarias. Puede tratarse de asignaturas o bien de requisitos (evaluables, por ejemplo, a través de la traducción de un texto de la disciplina en la que está formándose el estudiante) para cursar ciertas materias o tramos de la carrera. ${ }^{10}$ Por supuesto, no todas las carreras universitarias presentan esta exigencia de modo explícito. Sin embargo, está implícita en la obligatoriedad de lectura de bibliografía en lengua no española (y aun en la disponibilidad de esta última, con la ventaja comparativa que el acceso a ella conlleva). Acreditar dominio de otra lengua es casi siempre, además, condición necesaria para acceder a becas o pasantías en países extranjeros no hispanoparlantes, pero incluso en las evaluaciones de antecedentes para becas en el país, o en concursos para la cobertura de cargos docentes, lo que suele llamarse conocimiento de idiomas comúnmente se pondera. En otras palabras: el sistema universitario insta a quienes participan en él a que dominen alguna(s) lengua(s) de las llamadas extranjera(s), sea que la institución de que se trate ofrezca de modo formal la posibilidad de ese acceso, curricular o extracurricularmente, o no.

En cualquiera de estos casos, otros actores que conocen la exigencia señalada y ofrecen servicios vinculados con ella pueden publicitarlos en el ámbito universitario: centros privados de enseñanza de idiomas o profesores particulares pueden promocionar sus clases, tanto "llenando huecos" en relación con la oferta institucional como superponiéndose a esa oferta.

Dado que unos y otros suponen (y/o hacen suponer) que lo que ofrecen es deseable para los alumnos universitarios, en este artículo nos ocuparemos, a través del estudio de la publicitación de la oferta en un mismo -acotado- paisaje lingüístico institucional y en un mismo corte sincrónico, de apreciar comparativamente en ambas direcciones (top-down y bottom-up) a qué lenguas extranjeras se atiende y qué beneficios se invocan como motivaciones posibles para incentivar su estudio. Complementariamente, nos ocuparemos de analizar además la aparición o no de emisiones en esas mismas lenguas en la publicitación respectiva.

\section{Datos del paisaje institucional universitario de Bahía Blanca}

La primera observación que nuestros datos permiten es que, en el caso que analizamos, la publicitación que nos interesa corresponde, en la dirección top-down, exclusivamente a ofrecimientos

9. Los primeros resultados del procesamiento de los datos recogidos fueron presentados en el Congresso da Associação de Linguística e Filologia da América Latina, desarrollado en la Universidade Federal da Paraíba en octubre de 2018 (Hipperdinger, 2018).

10. En el caso de carreras de posgrado, la demostración del cumplimiento de tal exigencia puede constituir incluso un requisito de ingreso. 
extracurriculares. Toda la cartelería relevada, así, se orienta a "público-no-cautivo", por lo que el uso de estrategias de persuasión puede identificarse como necesidad en todos los casos (i.e. no solo en la dirección bottom-up, en la que tal necesidad puede asumirse a priori). Esto simplifica la comparación, consiguientemente, en la medida en que la limita a la dirección, sin necesidad de subclasificación alguna.

La segunda observación es que las lenguas extranjeras para las que se ofrecen clases son básicamente las mismas en ambas direcciones: inglés, italiano, francés, alemán y chino; ${ }^{11}$ en dirección top-down se ofrecen, además, portugués y griego moderno. Las siguientes fotografías muestran el ofrecimiento de clases de una misma lengua (el chino) en ambas direcciones:

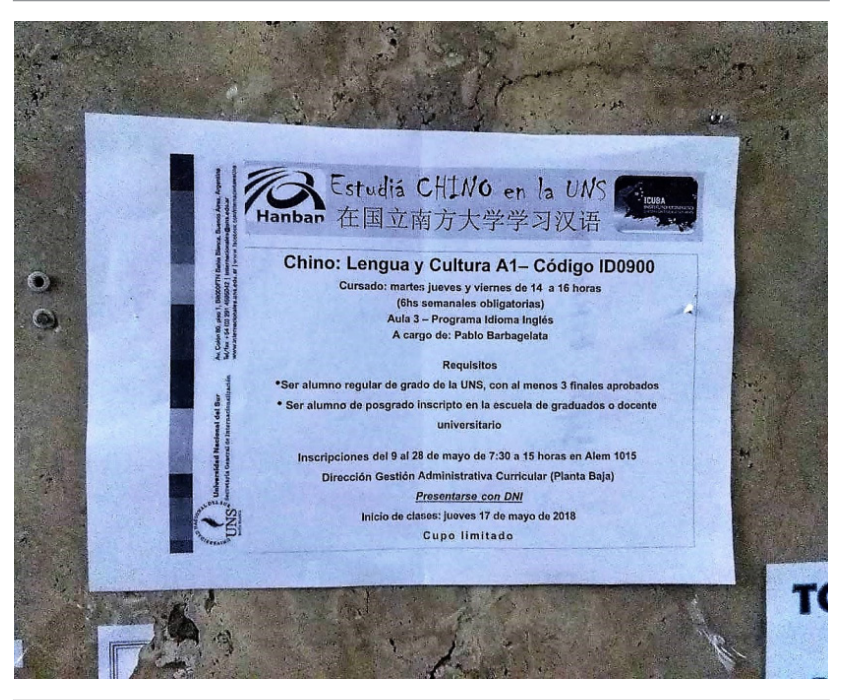

Fotografía 1. Ofrecimiento de clases de chino por la Universidad Nacional del Sur

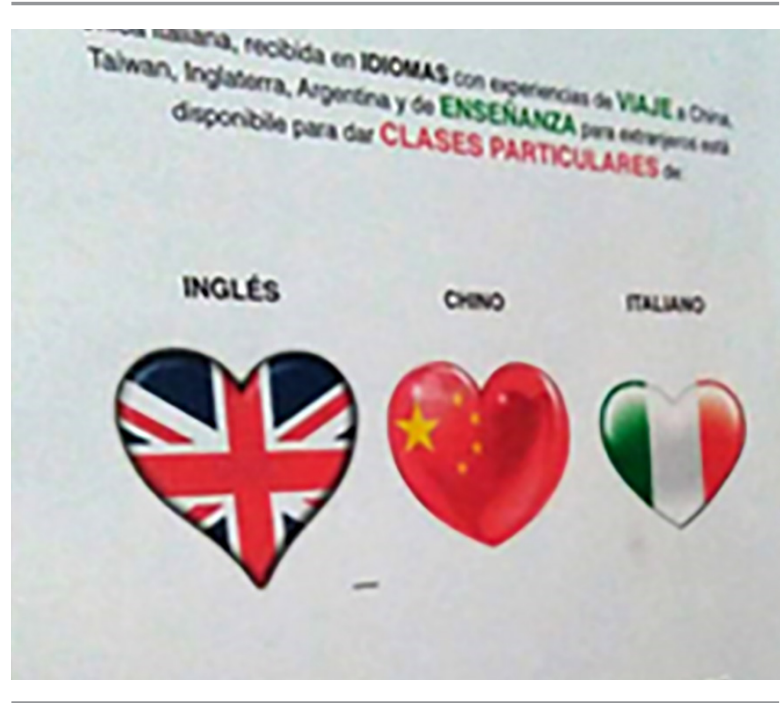

Fotografía 2. Ofrecimiento de clases de chino (y de inglés e italiano) por una profesora particular, publicitado en la Universidad Nacional del Sur ${ }^{12}$

Las lenguas referidas pueden ordenarse por frecuencia en lo que respecta a la publicitación de clases que en ellas registramos por distintos actores, o por el mismo para dos tipos diferentes de destinatarios intencionados. Como concreción de esta última posibilidad, no obstante, encontramos un único caso, que es el de cursos de alemán promocionados por la Sociedad Escolar Alemana (con convenio vigente con la Universidad Nacional del Sur - v. nota 11-) solo para alumnos universitarios o para cualesquiera interesados, como lo muestran las fotografías que siguen:

11. Consideramos de ofrecimiento oficial el de cursos de francés y alemán dictados, respectivamente, en la Alianza Francesa y la Sociedad Escolar Alemana, cuando lo son en el marco de convenios específicos sostenidos con la Universidad a tal efecto.

12. Hemos quitado los datos de contacto de las fotografías de anuncios de personas particulares. 


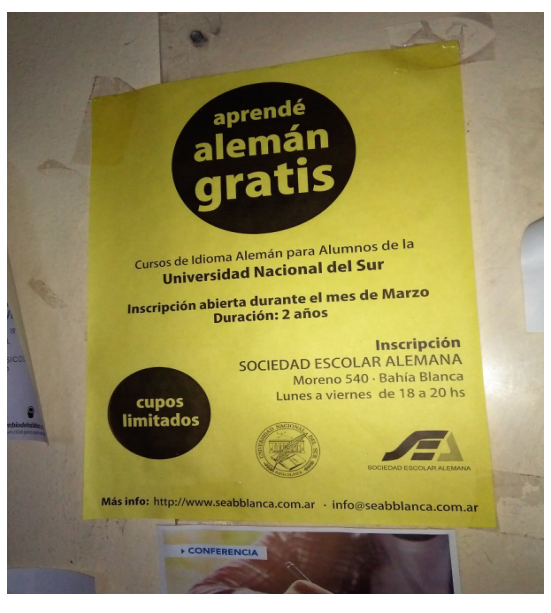

Fotografía 3. Ofrecimiento de clases de alemán para alumnos de la Universidad Nacional del Sur

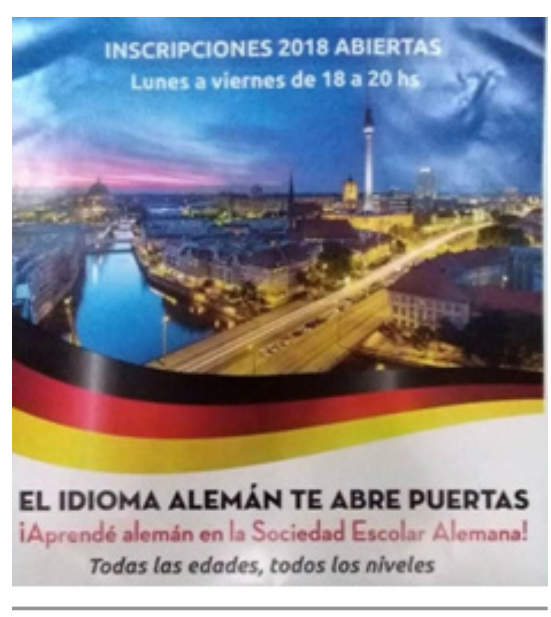

Fotografía 4. Ofrecimiento de clases de alemán para cualesquiera interesados, publicitado en la Universidad Nacional del Sur ${ }^{13}$

El orden de frecuencia referido es el siguiente:

Inglés (45.83\%)

Italiano $(16.67 \%)$

Francés (12.5\%)

Alemán (8.33\%) - Chino $(8.33 \%)^{14}$

Griego moderno (4.17\%) - Portugués (4.17\%)

En la cartelería se aprecia además, en ocasiones, que el ofrecimiento de un mismo emisor a unos mismos destinatarios puede asumir formas alternativas, como en el siguiente caso:

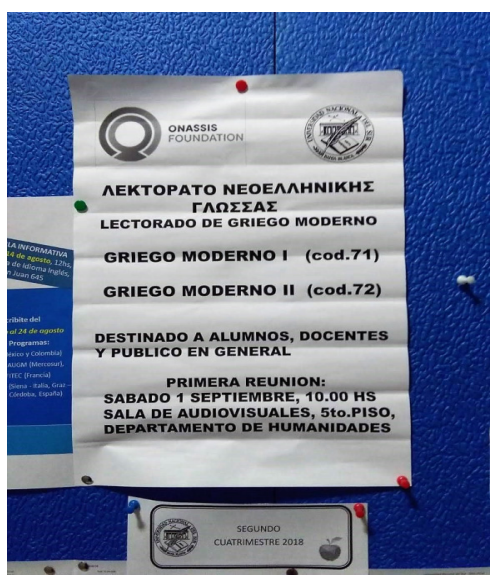

Fotografía 5

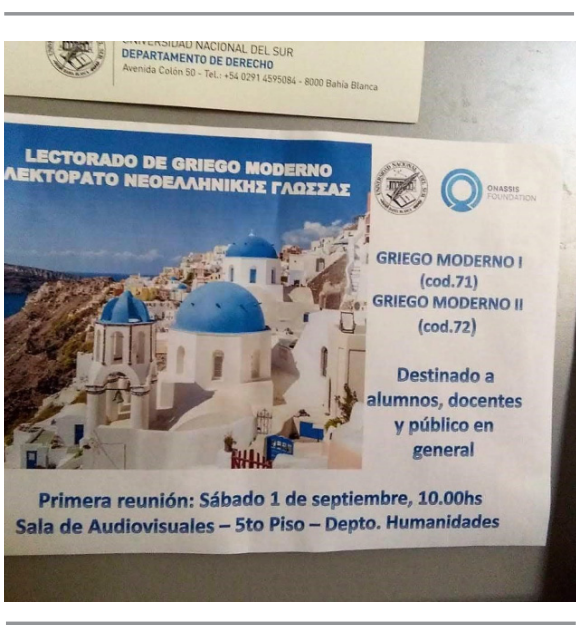

Fotografía 6

13. En este caso, la Fotografía 4 muestra (en el marco del convenio mencionado) la dirección top-down; la Fotografía 5, en cambio, corresponde a la dirección bottom-up, aunque se trate de un mismo emisor.

14. A igual frecuencia, seguimos un criterio alfabético. 
Con independencia de los detalles, y según puede apreciarse, lo que hemos consignado hasta aquí indica principalmente la restricción de las opciones y la preeminencia, entre esas opciones, del inglés.

En cuanto a las estrategias de promoción, la principal observación es que no siempre se invoca un beneficio, o no de modo transparente. La Fotografía 4 muestra una formulación explícita (aunque elíptica) respecto de un beneficio: "El idioma alemán te abre puertas". En cambio, el banner a través del cual se anuncia el ofrecimiento de chino y portugués (además de español para extranjeros) en la Secretaría General de Internacionalización -hoy Subsecretaría de Internacionalización- de la Universidad Nacional del Sur no invoca, respecto del aprendizaje de esas lenguas, beneficio alguno:

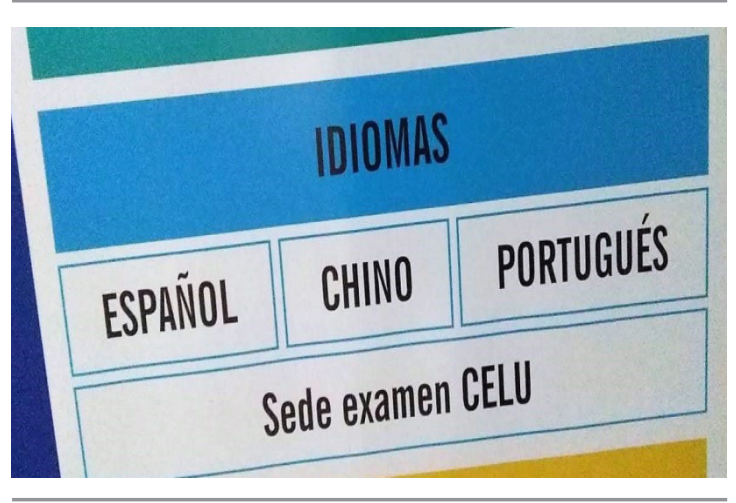

Fotografía 7

La Fotografía 4 sugiere, además, otro beneficio: el de conocer la lengua alemana para viajar, como se implica en la imagen panorámica de Berlín que domina el afiche. La vista de Santorini que se aprecia en la Fotografía 6 se corresponde con la misma estrategia. En igual sentido se orientan las banderas presentadas en el anuncio que se recoge en la Fotografía 2. Una combinación de imagen con los colores de una bandera se aprecia en el afiche siguiente:

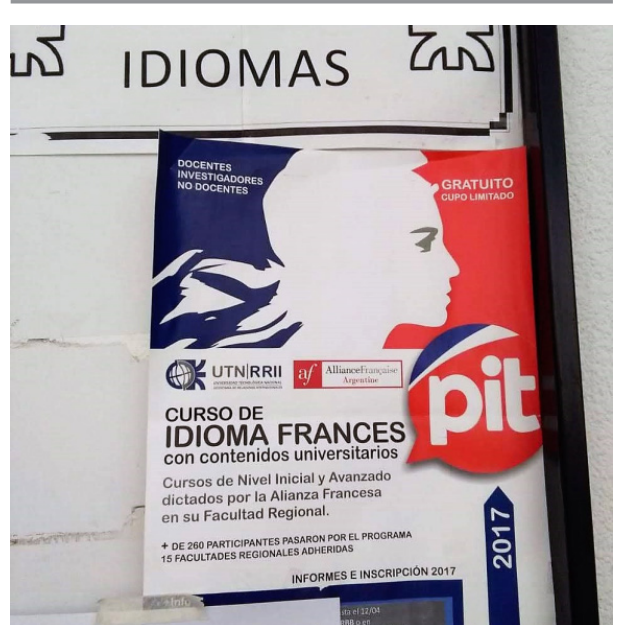

Fotografía 8. Sección de cartelería correspondiente al Centro de Idiomas de la Universidad Tecnológica Nacional 
Una combinación también, pero de formulación lingüística y sugerencia por la vía de la imagen, se puede apreciar en el siguiente anuncio:

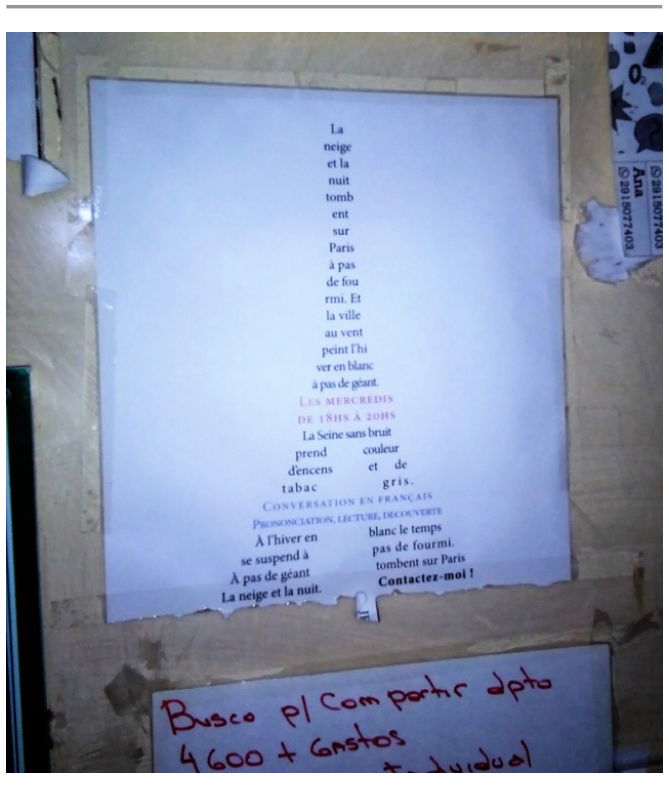

Fotografía 9. Ofrecimiento de clases particulares de francés, publicitado en la Universidad Nacional del Sur

En este último anuncio, la imagen de la Tour Eiffel ha sido construida con los versos de un poema de Pierre Coran, Paris blanc, en que se intercala el texto del ofrecimiento. Este anuncio es de interés, por consiguiente, no solo por su delicado equilibrio entre explicitación y sugerencia sino también porque, para promocionar el ofrecimiento de clases en una lengua extranjera, usa esa lengua. Lo hace, además, tanto de modo simbólico como de modo comunicativo, mientras que la recurrencia a la escritura china en el anuncio que puede verse en la Fotografía 1, o a la escritura griega en las Fotografías 5 y 6, tienen carácter exclusivamente simbólico (la lengua de comunicación es el español, y en la otra lengua solo se repite parte de lo que se dice en español).

En síntesis, los beneficios que encontramos invocados para incentivar el deseo de aprender las distintas lenguas extranjeras lo son o bien a través de una formulación lingüística, o a través de la sugerencia posibilitada por una imagen que emblematiza el país (o uno de los países) en que se habla la lengua de que se trata.

Los beneficios expresamente enunciados, sin embargo, los encontramos únicamente (con la sola excepción de las "puertas" que abre el alemán) en el caso de anuncios de clases de inglés. Por otra parte (y frente a la indeterminación de las "puertas" referidas), estos anuncios de clases de inglés apuntan a objetivos concretos: preparar a los alumnos universitarios para rendir exámenes de suficiencia que son requisito en sus carreras, prepararlos para exámenes internacionales requeridos para becas en el exterior, etc. Lo muestran, por ejemplo, los anuncios siguientes: 


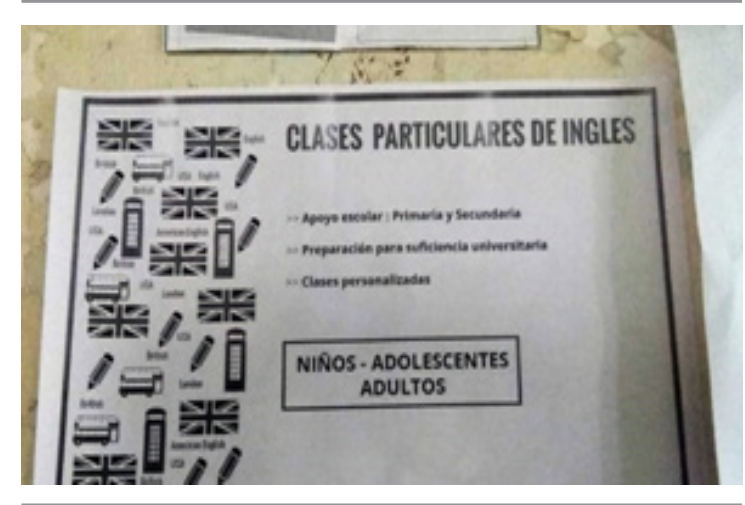

Fotografía 10

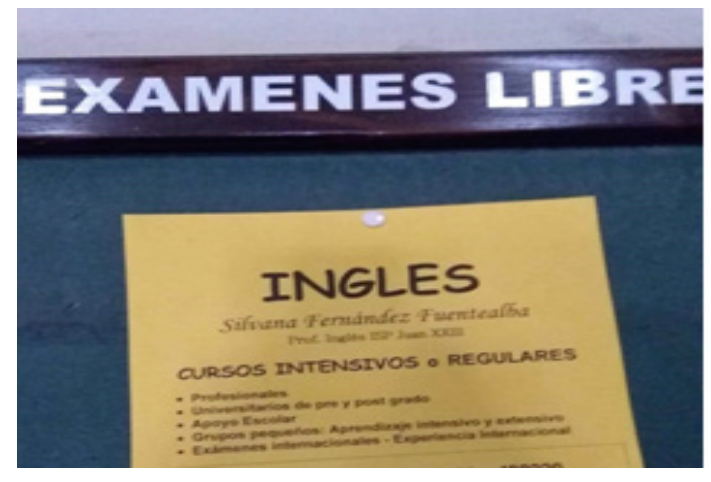

Fotografía 11

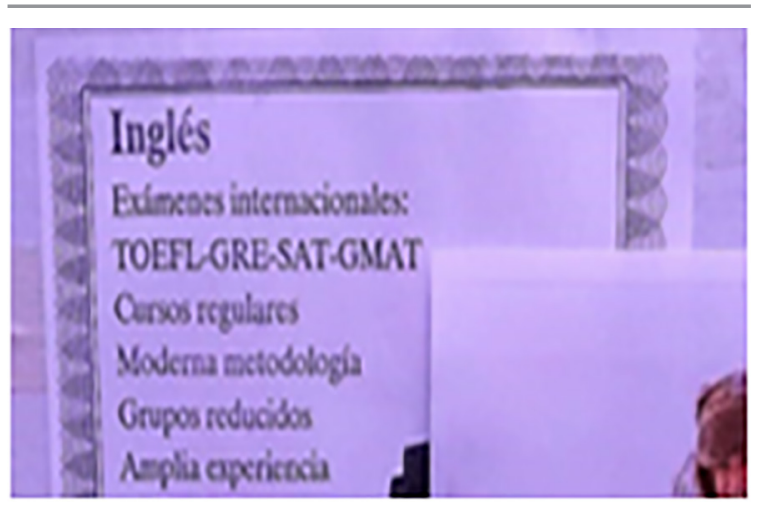

Fotografía 12

En contraposición, en anuncios de clases de inglés las únicas imágenes de carácter emblemático que encontramos empleadas son las que muestran las Fotografías 2 y $10 .{ }^{15}$ Por el contrario (y con la única excepción del portugués), encontramos tales imágenes en anuncios de todas las demás lenguas publicitadas: en dos tercios de los anuncios de clases de francés y en la mitad de los anuncios correspondientes a las lenguas restantes. Un interés por hacer más atractivos los anuncios de las lenguas que no son el inglés puede inferirse de ello: si a las demás lenguas "hay que venderlas", el inglés "se vende solo".

Por último, en la cartelería relevada encontramos inscripciones en la lengua de que se trata solamente en los casos del inglés, el francés, el chino y el griego moderno.

En estos dos últimos casos, según llevamos dicho, los usos tienen un carácter exclusivamente simbólico. También lo tienen las inscripciones en inglés en el único anuncio en que las constatamos, y que muestra la Fotografía 10, ${ }^{16}$ aunque es frecuente en los anuncios académicos de oportunidades para los estudiantes universitarios que el inglés sí se emplee como lengua de comunicación (incluso cuando

15. Nótese que el anuncio que recoge la Fotografía 12 remite a estudios y graduación universitaria, no a viajes ni nacionalidades.

16. El empleo no solo simbólico del francés que muestra la Fotografía 9 es, por lo tanto, atípico y responde a previsiones sobre la "audiencia" (Bell, 1984) preseleccionada: el uso comunicativo del francés en el aviso es un indicador de que las clases están dirigidas solamente a personas con conocimiento previo de esa lengua. 
otra lengua pueda ser -también- requerida para la oportunidad aludida), como se ve en los anuncios siguientes:

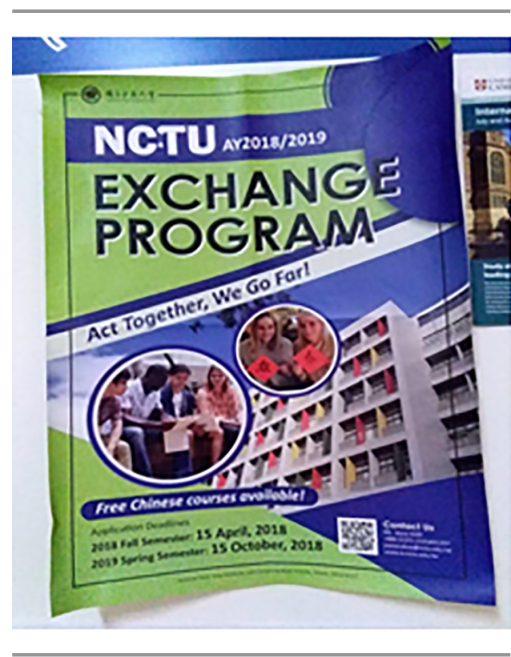

Fotografía 13

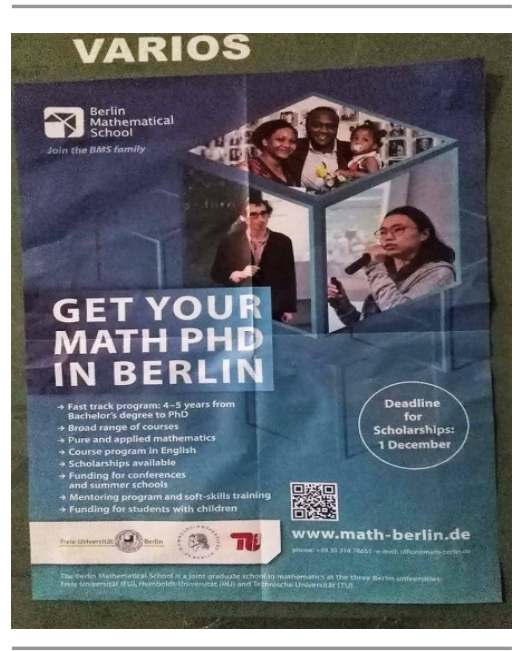

Fotografía 14

En lo que respecta al uso de la propia lengua promocionada en la cartelería que nos interesa, así, puede hacerse una observación general sobre su irrelevancia comunicativa y su regular valor simbólico, por un lado, y por otro lado sobre la especialmente escasa recurrencia al inglés en anuncios de clases de inglés, menos frecuente que en el conjunto de las demás y llamativa, especialmente, por el contraste que con el uso que del inglés se aprecia, en casos como los que muestran las dos últimas fotografías, en la cartelería universitaria analizada.

\section{Conclusiones}

La exposición a la que hemos procedido muestra un conjunto de resultados, que corresponde confrontar con los que se obtengan sobre muestras empíricas más amplias pero que presentan interés intrínseco por su posible interpretación como marcadores, en el sentido en que usan este término Landry y Bourhis (1997):

1) La generalizada coincidencia que encontramos en ambas direcciones pone de manifiesto la restricción de las opciones y la centralidad del inglés, ligadas a la prevalencia de su valor instrumental comparativo entre las motivaciones invocadas.

2) Aunque ese valor instrumental no está necesariamente ausente en la promoción de otras lenguas, o bien se lo invoca de modo inespecífico o bien se lo orienta a beneficios ligados preeminentemente a la movilidad: la conveniencia de dominar esas lenguas para "conocer el mundo"; esta posibilidad asume per se un valor simbólico (de cosmopolitismo, de apertura a la complejidad intercultural), que puede distinguirse de la instrumentalidad (aun cuando pueda concurrir con ella) en el rango, más acotado, de los intereses específicamente académicos.

3) Por último, es compatible con esas generalizaciones la de que el uso de la lengua promocionada en la cartelería relativa a su promoción, que regularmente no tiene valor comunicativo sino solo 
simbólico, se aprecie más comúnmente cuando el valor simbólico de la lengua es (también) invocado que cuando se enfoca (en exclusividad o preeminentemente) su valor instrumental.

Las diferencias apreciables en nuestros datos entre las direcciones top-down y bottom-up, en cualquiera de estos aspectos, son irrelevantes, si bien ello no puede desligarse del hecho de que se haya tratado exclusivamente de oferta extracurricular. En ambas direcciones, así, se "venden" lenguas, pero en ambas una (el inglés) no solo es comparativamente más "vendida" sino que se confía, en las estrategias de presentación, en que (principalmente sobre la base del general reconocimiento de la incomparabilidad de su valor instrumental con el de cualquier otra lengua extranjera) "se vende sola". Las representaciones sociales extendidas que hacen posible esa confianza, a su vez, son inseparables de la promoción formalizada curricularmente, si bien no puede suponérselas una mera derivación de ella.

Aunque ese interjuego queda por analizarse, el de las voces oficiales o no en la oferta opcional que analizamos muestra consensos que constituyen un buen punto de partida para ese estudio ulterior, tanto como muestra que, en la trama de recursos heurísticos idóneos para llevarlo a cabo, la atención a este tipo de paisaje lingüístico puede proveer datos de especial utilidad no solo en el plano glotopolítico (sea respecto de políticas institucionales explícitas cuanto de lo que es socialmente tácito respecto de las lenguas cuyo conocimiento se insta a sumar) sino también, más "de cerca", respecto de las estrategias que se ponen en juego.

Es en este último sentido, que tan bien evidencia que esta forma de espacialidad del lenguaje es de atención sociolingüística inexcusable, que los aportes de detalle contribuyen a robustecer la base empírica de una nueva summa del conocimiento sobre el español de la Argentina, cuya misma necesidad hace prever que seguramente se publicará, aunque se haga esperar.

\section{Referencias bibliográficas}

Albizu Echeipia, L. (2016). Análisis del paisaje lingüistico en un centro de educación secundaria: un camino bacia la aceptación de la diversidad lingüistica. Navarra, Universidad Pública de Navarra.

Bein, R. (2012). La política lingüistica respecto de las lenguas extranjeras en la Argentina a partir de 1993. Viena, Universität Wien.

Bell, A. (1984). Language style as audience design. Language in Society, 13 (2), 145-204.

Ben-Rafael, E., Shohamy, E., Amara, M. H. y Trumper-Hecht, N. (2006). Linguistic landscape as symbolic construction of the public space: The case of Israel. En Gorter, D. (ed.), Linguistic Landscape: A New Approach to Multilingualism (pp. 7-30). Clevedon, Multilingual Matters.

Blommaert, J. (2013). Ethnography, Superdiversity and Linguistic Landscapes. Chronicles of Complexity. Bristol, Multilingual Matters.

Blommaert, J. y Maly, I. (2014). Ethnographic linguistic landscape analysis and social change: A case study. Working Papers in Urban Language E Literacies, 133, 2-20. 
Bonnin, J. E. y Unamuno, V. (coords.) (2019). Paisajes lingüísticos en el mundo hispánico (Dossier). Signo É Seña, 35, 1-106.

Corbeil, J.-C. (1980). L’aménagement linguistique du Québec. Montréal, Guérin.

Fontanella de Weinberg, M. B. (1979). Dinámica social de un cambio lingüístico. La reestructuración de las palatales en el español bonaerense. Ciudad de México, Universidad Nacional Autónoma de México.

Fontanella de Weinberg, M. B. (coord.) (2000). El español de la Argentina y sus variedades regionales. Buenos Aires, Edicial.

Gorter, D. (2006). Introduction: The study of the linguistic landscape as a new approach to multilingualism. International Journal of Multilingualism, 3 (1), 1-6.

Gorter, D. y Cenoz, J. (2015). Linguistic landscapes inside multilingual schools. En Spolsky, B., InbarLourie, O. y Tannenbaum, M. (eds.), Challenges for Language Education and Policy: Making Space for People (pp. 151-169). New York, Routledge Publishers.

Griffin, J. (2004). The presence of written English on the streets of Rome. English Today, 20 (2), 3-7 y 47.

Hanauer, D. (2010). Laboratory identity: A linguistic landscape analysis of personalized space within a microbiology laboratory. Critical Inquiry in Language Studies, 7 (2-3), 152-172.

Hipperdinger, Y. (2018). La promoción de las lenguas extranjeras en el ámbito de la educación superior. Datos del paisaje lingüístico institucional de Bahía Blanca. Congresso da Associação de Linguística e Filologia da América Latina (ALFAL), João Pessoa, ALFAL-Universidade Federal da Paraíba.

Hipperdinger, Y. (ed.) (2019). Español y otras lenguas en el área dialectal bonaerense del español de la Argentina. Bahía Blanca, EdiUNS.

Ivković, D. y Lotherington, H. (2009). Multilingualism in cyberspace: Conceptualising the virtual linguistic landscape. International Journal of Multilingualism, 6 (1), 17-36.

Jaworski, A. y Thurlow, C. (2010). Introducing Semiotic Landscapes. En Jaworski, A. y Thurlow, C. (eds.), Semiotic Landscapes: Language, Image, Space (pp.1-40). London, Continuum.

Landry, R. y Bourhis, R. (1997). Linguistic landscape and ethnolinguistic vitality: An empirical study. Journal of Language and Social Psychology, 16 (1), 23-49.

Leimgruber, J. (2019). Montreal's linguistic landscape: Instances of top-down and bottom-up language planning. En Castillo Lluch, M., Kailuweit, R. y Pusch, C. (eds.), Linguistic Landscape Studies: The French Connection (pp. 163-174). Freiburg im Breisgau, Rombach.

Pons Rodríguez, L. (2012). El paisaje lingüistico de Sevilla. Lenguas y variedades en el escenario urbano hispalense. Sevilla, Servicio de Archivo y Publicaciones de la Diputación de Sevilla.

Pozzo, M. I. (2009). La enseñanza de lenguas extranjeras en Argentina. Diálogos latinoamericanos, 15, s/p.

Rámila Díaz, N. (2015). El paisaje lingüístico o la construcción de un espacio híbrido en el Instituto Cervantes de París. Estudios interlingüísticos, 3, 89-104. 
Rosenbaum, Y., Nadel, E., Cooper, R. L. y Fishman, J. A. (1977). English on Keren Kayemet street. En Fishman, J. A., Cooper, R. L. y Conrad, A. W. (eds.), The spread of English (pp. 179-196). Rowley, Newbury House.

Scollon, R. y Wong Scollon, S. (2003). Discourses in Place. Language in the Material World. London, Routledge.

Shohamy, E.y Gorter,D. (eds.) (2009). Linguistic Landscape. Expanding the Scenery. London, Routledge.

Van Mensel, L., Vandenbroucke, M. y Blackwood, R. (2016). Linguistic landscapes. En García, O., Flores, N. y Spotti, M. (eds.), Oxford Handbook of Language and Society (pp. 423-449). Oxford, Oxford University Press.

Verdoot, A. (1979). La protection des droits de l'bomme dans les états plurilingües. Paris, Fernand Nattan. Vidal de Battini, B. (1964). El español de la Argentina. Buenos Aires, Consejo Nacional de Educación.

Wee,L.(2021). The Communicative Linguistic Landscape: Production Formats and Designed Environments. London, Routledge.

*Yolanda Hipperdinger es Licenciada y Doctora en Letras con orientación en Lingüística. Es Investigadora Independiente del CONICET y Profesora Asociada ordinaria en el Departamento de Humanidades de la Universidad Nacional del Sur. Se ha especializado en sociolingüística y contacto lingüístico, y en relación con esos campos de estudio ha ofrecido conferencias, cursos y seminarios en diversas universidades del país y del exterior. Entre sus publicaciones se cuentan libros, capítulos, artículos y críticas en revistas especializadas, publicados en distintos países americanos y europeos. Forma parte del cuerpo docente de varias carreras de posgrado y, desde el año 2002, dirige proyectos grupales de investigación acreditados. Entre 2014 y 2016 ha sido Presidenta de la Sociedad Argentina de Lingüística.

RECIBIDO: $1 / 11 / 2020$

ACEPTADO: $1 / 12 / 2020$ 